Araştırma Makalesi / Research Article

Elektrik Elektronik Mühendisliği /

Electrical Electronic Engineering
Iğdır Üniversitesi Fen Bilimleri Enstitüsü Dergisi, 10(1): 118-127, 2020 Journal of the Institute of Science and Technology, 10(1): 118-127, 2020

\title{
Reliable Video Multicasting over WLANs
}

\section{Berna BULUT ${ }^{1 *}$}

\begin{abstract}
As is known reliable multicast transmission is not addressed in WLANs. Multicast packets are sent over wireless channels without using medium access control (MAC) layer automatic repeat request (ARQ) mechanism. Therefore, packet error rates will be very high. This paper investigates reliable video multicasting, which became a very popular application, over WLANs. To this end, application layer forward error correction (AL-FEC) codes based on Raptor Q can be used as a means to provide reliable delivery of the multicast video encoded with the H.264/Advanced video coding (AVC) codec. Since video streaming applications have very stringent Quality of Service requirements (low delay and error free transmission of packets), the Raptor Q and the H.264/AVC parameters have to be determined depending on the QoS of the video. Therefore, in this work an advanced cross-layer simulator was developed to analyse the performance of the end-to-end system. Simulation results show that using AL-FEC significantly improve the received video quality, i.e., the mean peak signal to noise ratio (PSNR) is improved over $34 \mathrm{~dB}$. Further that it is shown that frequently inserting intra-frame results in poor video quality. Therefore, it is suggested to send intra frame at longer intervals.
\end{abstract}

Keywords: AL-FEC, Multicast, Video streaming, H.264/AVC, WLAN

\footnotetext{
${ }^{1}$ Berna BULUT (Orcid ID: 0000-0001-5988-4718), Kabaoğlu Mahallesi, Prof. Baki Komsuoglu Bulvar1, No:515EU. Kocaeli, Turkey

*Corresponding author: Berna Bulut, e-mail: berna.bulut1@ hotmail.com
}

Geliş tarihi / Received: 19-11-2019

Kabul tarihi / Accepted: 28-11-2019 


\section{INTRODUCTION}

The use of WLANs for multimedia distribution to mobile terminals such as phones is increasing. Especially for some evets such as sports, concerts many users want to connect to the network at the same time in order to acquire the data. When the transmission scheme is unicast, the same content is delivered to each user separately. This wastes valuable networks and radio resources since the available bandwidth in WLANs is limited which is also shared between the users thus when the number of users get higher there will be a bandwidth shortage in the network. Bandwidth limitation becomes severe when each unicast user also requests the transmitter to retransmit the lost packets via using medium access control (MAC) layer automatic repeat request (ARQ) mechanism. In such scenarios where multiple users want to access the same data simultaneously, multicast transmission is one of the best solutions to efficiently deliver such applications (e.g., live video) over wireless channels to mobile users. However, WLANs standard does not provide any solutions or schemes for reliable multicast transmission (IEEE Std 802.11, 2012). Multicast packets are transmitted over wireless channels without getting any feedbacks from users, i.e., there is no MAC layer ARQ packet retransmission mechanism. Therefore, in multicast/broadcast transmissions, high packet losses are observed.

Video streaming services cannot overcome high packet loss rates which lead serious degradation in the perceived video quality. To improve the performance of the multicast/broadcast schemes, in MBMS (3GPP TS 26.346 V8.0.0, 2008) application layer forward error correction (AL-FEC) based on Raptor codes is suggested to provide reliability for multicast/broadcast streaming and download delivery services. Since there is no ARQ mechanism in multicast/broadcast transmission, AL-FEC scheme implements sending redundant data along with the original data (packets) to allow the receiver (video decoder) to recover the corrupted source data (lost packets) by using the redundant ones.

To this end, some works have investigated the cross-layer optimisation frameworks in which the AL-FEC redundancy is adjusted depending on the selected physical layer modulation and coding schemes (MCSs) in order to provide reliable video streaming applications over unreliable wireless channels (Haratcherev et al., 2006; Van der Shaar and Turaga, 2007; Lee and Chung, 2008; Choi et al., 2014). However, multicast streaming of video to multiple users have additional challenges since each multicast user has unique channel condition. Thus, it is not very feasible to find system parameters depending on the quality of service (QoS) requirements of the applications that are optimal (provide good quality of experience (QoE)) for each multicast user. Only a number of works consider the specific needs of multicast video streaming over wireless networks (Ma et al., 2005; Afzal et al., 2006; Chen et al., 2007; Samokhina et al., 2008).

The H.264/Advanced video coding (AVC) standard (ITU-T Recommendation H.264, 2009) known as ITU-T H.264 is one of the most commonly implemented codecs today. H.264/AVC defines two layered architectures. These are network abstraction layer (NAL) and video coding layer (VCL). VCL stands for the encoded video data (i.e., bit sequence), the NAL converts the encoded video stream (bits) into packets (packetization), which are called NAL units (NALUs), to be delivered over an underlying packet-based network (Stockhammer et al., 2003; Wiegand, 2003). A NALU can be either inter-frame coded (P) or intra-frame coded (I frame) (Stockhammer et al., 2003). The I frames do not depend on $\mathrm{P}$ frames, however, $\mathrm{P}$ frames do since these frames are predicted from other $\mathrm{P}$ and I frames. In a video squence, some frames at specific periods are intra-frame coded because I frames enable resynchronisation and stop propagation of errors in the video sequence (Ferre, 2006).

Therefore, in this paper a cross-layer framework which considers not just the AL-FEC but also the H.264 codec properties (specifically I frame interval) at the application layer for reliable and scalable 
multicast video streaming over WLANs is presented. In this work, the latest Raptor codes namely Raptor $\mathrm{Q}$ is considered. A detailed cross-layer simulator was developed to evaluate the end-o-end system performance for different cross-layers` parameters.

\section{MATERIALS AND METHODS}

A detailed cross-layer simulator was developed in MATLAB to evaluate the end-to-end system performance for a large set of cross-layer parameters. The cross-layer simulator consists of four modular subsystems: 1) the H.264/AVC video codec, 2) RQ, 3) MAC-Physical layers of WLANs, and 4) the channel simulators. Each of these simulators is developed separately to overcome the complexity and the computation time.

\section{H.264/AVC codec}

The H.264/AVC simulator can model the streaming of any video sequence from transmitter to user end over the physical and MAC layers of WLANs to evaluate the quality of the received video. According to the H.264/AVC standard, the video encoder encapsulates video stream into NALUs. In the simulations, it is considered that one NALU is also mapped into one RTP/UDP packet which has a fixed size. The H.264/AVC video codec enables encoding the video sequence with different configurations such as different bit rates, packet (NALU) sizes, frame rates, I frame periods (intervals) etc.

At the H.264/AVC decoder, an error concealment schemes defined in the H.264/AVC standard called Previous Frame Copy is used (H.264/AVC reference software, 2015). A reference H.264/AVC video decoder is available in (H.264/AVC reference software, 2015) is implemented for decoding the video sequences. At the receiver, the video quality is measured in terms of peak signal to noise ratio (PSNR) per frame and the average PSNR over the entire video sequence (over all frames of the video). PSNR is a statistical measure frequently used in literature to quantify the received video quality. The PSNR for a video frame is calculated by taking the mean square error (MSE) of the received video frame and comparing it with a reference frame which is generated error free at the encoder.

\section{AL-FEC simulator}

Raptor codes are rateless application layer forward error correction codes implemented across packets to protect the transmitted data against packet losses (Shokrollahi, 2006; Shokrollahi and Luby, 2011). At the application layer, the incoming RTP/UDP packets are collected by Raptor Q encoder to construct the source blocks each consists of $K$ source packets (symbols) with $T$ bytes and then generates $N$ encoded symbols with $T$ bytes from each block. Since the Raptor Q codes are systematic codes, the first encoding symbols of $N$ encoded symbols are the original $K$ source symbols and the remaining $R$ symbols of $N$ symbols are called the repair symbols $(N=K+R)$. Raptor Q code rate is written as as $C R=K / N=K /(K+R)$.

At the receiver side, the Raptor Q decoder waits upon to receive all the UDP packets belong to a given source block. At the decoder, when the overall acquired packets (source and repair symbols) for a given source block is $K^{\prime} \geq(\varepsilon+1) K$, then the Raptor Q decoder can decode the block with high probability, i.e., all source packets of the source block are reconstructed and conveyed to application layer. However, if the decoding could not successfully decode the source block, then only acquired original source UDP packets are delivered to the H.264/AVC video codec.

\section{MAC-Physical layer simulator}

Effective signal-to-noise-plus-interference ratio (SINR) mapping (ESM) physical layer abstraction model called as the received bit mutual information (RBIR) (Wan et al., 2006), is implemented to generate the packet error rate (PER) $P e$. In ESM model, a block of OFDM subcarrier SINRs is translated 
into a single effective SINR (ESINR) value. This ESINR value is used to calculate the PER values for any given MCS by using a non-faded PER versus SINR look up table. This SINR look up table is created by performing bit level simulations for an additive white gaussian noise (AWGN) channel. The MCSs for the WLANs`20 MHz channel bandwidth with a $400 \mathrm{~ns}$ guard interval (GI) (IEEE Std 802.11, 2012) are used in the RBIR simulator for a multiple input multiple output (MIMO) system that as seen in Table 1.

The MAC-Physical layer simulator, which is based on the distributed coordination function (DCF) with basic access scheme as stated in WLAN standard (IEEE Std 802.11, 2012), replicates the packet loss trace for a sequence of NALUs. Since the transmission model is multicast MAC layer ARQ is not implemented. It is considered that one NALU is encapsulated into one RTP/UDP/IP packet since multicast packets in WLANs is not fragmented at the MAC. Therefore, there is a one-to-one mapping between NALUs and physical layer protocol data units (PPDU), i.e., PERs at the physical layer show the NALU loss rates at the video decoder. Simulation is executed for all given MCS modes and Raptor $\mathrm{Q}$ code rates.

\section{Channel model}

The system performance is evaluated for a mobile users in an outdoor environment. The TGn channel model F defined in (Erceg et al., 2004) is considered in this work. This channel model typically represents a non-line-of-sight (NLOS) large outdoor scenario with an root mean square (RMS) delay spread of $150 \mathrm{~ns}$. It is assumed that base station (BS) and mobile station (MS) operate in the $2.4 \mathrm{GHz}$ band are equipped with two transmit and receive antennas. Thus, they can provide up to 2 spatial streams. The transmit power is $20 \mathrm{dBm}$. BS and MS were placed at $2.5 \mathrm{~m}$ and $1 \mathrm{~m}$ above the ground respectively. The parameters used in the channel model is summarised in Table 1.

Table 1. Simulation parameters

\begin{tabular}{ll}
\hline Parameter & Value \\
\hline Raptor symbol size, $T$ & 1400 Bytes \\
Source block length, $K$ & 100,200 \\
Video bit rate & $4 \mathrm{Mbps}$ \\
I frame interval/period & 5,25 \\
MCS mode & MCS 9 (QPSK 1/2) \\
BS height & $2.5 \mathrm{~m}$ \\
MS height & $1 \mathrm{~m}$ \\
Number of antennas at the BS and MS & $2($ up to two streams) \\
Channel frequency & $2.4 \mathrm{GHz}$ \\
Channel bandwidth & $20 \mathrm{MHz}$ \\
GI & $400 \mathrm{~ns}$ \\
\hline
\end{tabular}

\section{RESULTS AND DISCUSSION}

To evaluate the system performance, the Rush hour video sequence with a resolution of 720p is used. The video was encoded with a bit rate $\left(V_{B R}\right)$ of $4 \mathrm{Mbps}$. The video sequence comprises of 200 frames encoded at 25 frames per second, and I frames are sent every 5 th and 25 th frame intervals. The total number of NALUs is 2942 and encapsulated in 2942 RTP/UDP packets. The video quality is evaluated in terms of PSNR for different Raptor Q source block sizes ( $K=100$ and $K=200)$, Raptor Q code rates $(C R=\{0.9,0.8,0.7\})$, I frame periods $\left(I_{F P}=5\right.$ and $\left.I_{F P}=25\right)$, and MCS 9 . 
Figure 1 shows the received packet trace (a packet can be either lost or received correctly) at the video decoder for transmitting 2942 NALUs over WLANs using MCS 9. It is seen that packet loss is quite bursty (consecutive packets are lost). Using the received packet trace in Figure 1, the block error rates at the Raptor Q decoder can be calculated for different source block sizes as seen in Figure 2 and Figure 3 which present the PER in each source block for $K=100$ and $K=200$. The total number of source block, $N B$, depends on the source block size, $K$, and the total number of NALUs, $N_{N A L U}$, since it calculated as $N B=\left\lceil\frac{N_{N A L U}}{K}\right\rceil$, where $\lceil x\rceil$ denotes the smaller integer greater than or equal to $x$. Therefore, the $N B$ values calculated for $K=100$ and $K=200$ are 30 and 15 respectively. As seen in Figure 2 and Figure 3 that the peak PER in a source block reduces when higher source block size $(K=200)$ is used, i.e., the peak PER when $K=100$ is 0.46 while for $K=200$ it is 0.245 . This means that the Raptor Q decoder need less repair symbols when the peak PER in a source block is small (higher source block size is used). Although, using higher source block size $(K=200)$ reduces the peak PER in a source block and hence the required repair symbols, it causes a constant longer Raptor Q encoding delay, $D$, which is calculated as $D=(K . T .8) / V_{B R}$, since the Raptor Q encoder waits to collect enough source packets (symbols) to form a source block consists of $K$ source symbols each with $T$ bytes. The Raptor Q decoder tries to recover the all source symbols in a source block which obviously depends on the PER in a source block and the Raptor Q code rate. If the decoder has enough repair symbols, then it is high likely to recover that source block and deliver all source packets to the video decoder otherwise only received source symbols (packets) will be delivered for that source block to the video decoder.

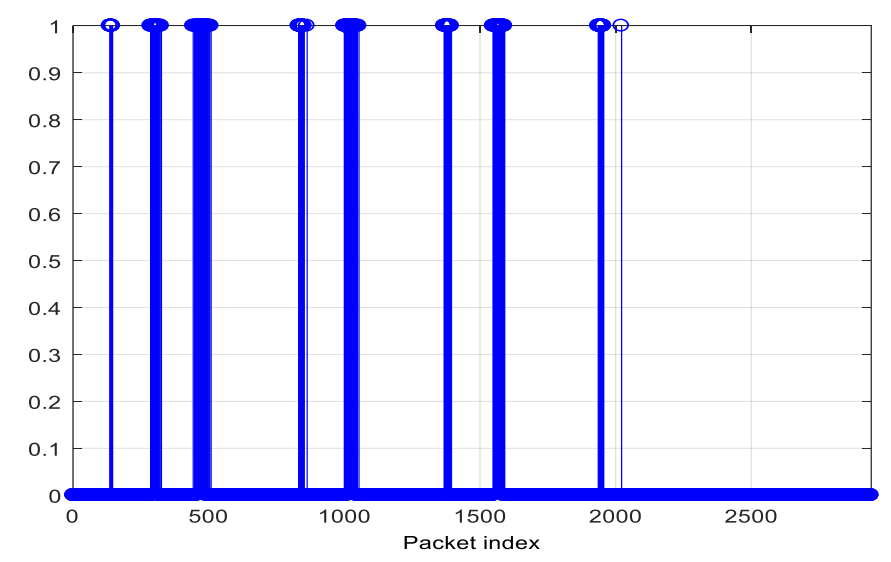

Figure 1. Received packet trace (1: lost packet, 0: received packet), MCS 9

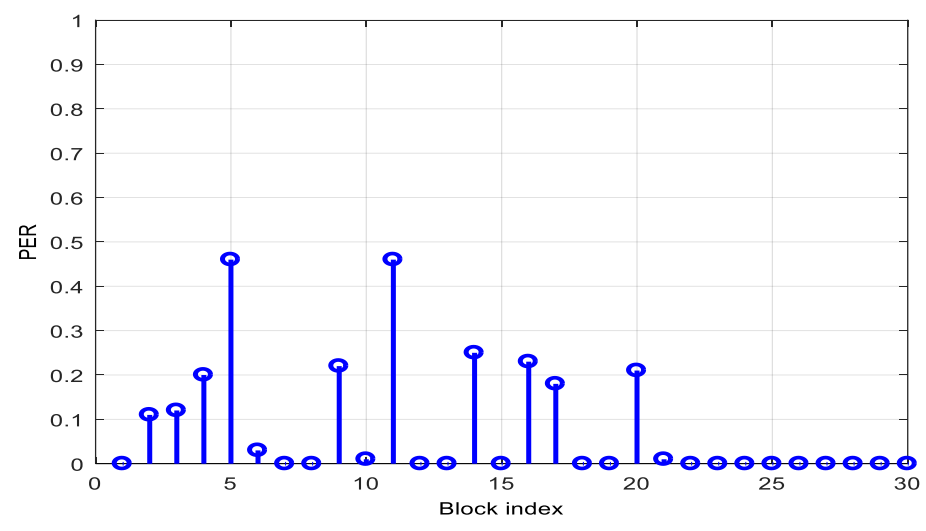

Figure 2. PER per Raptor Q source block, $K=100$ 


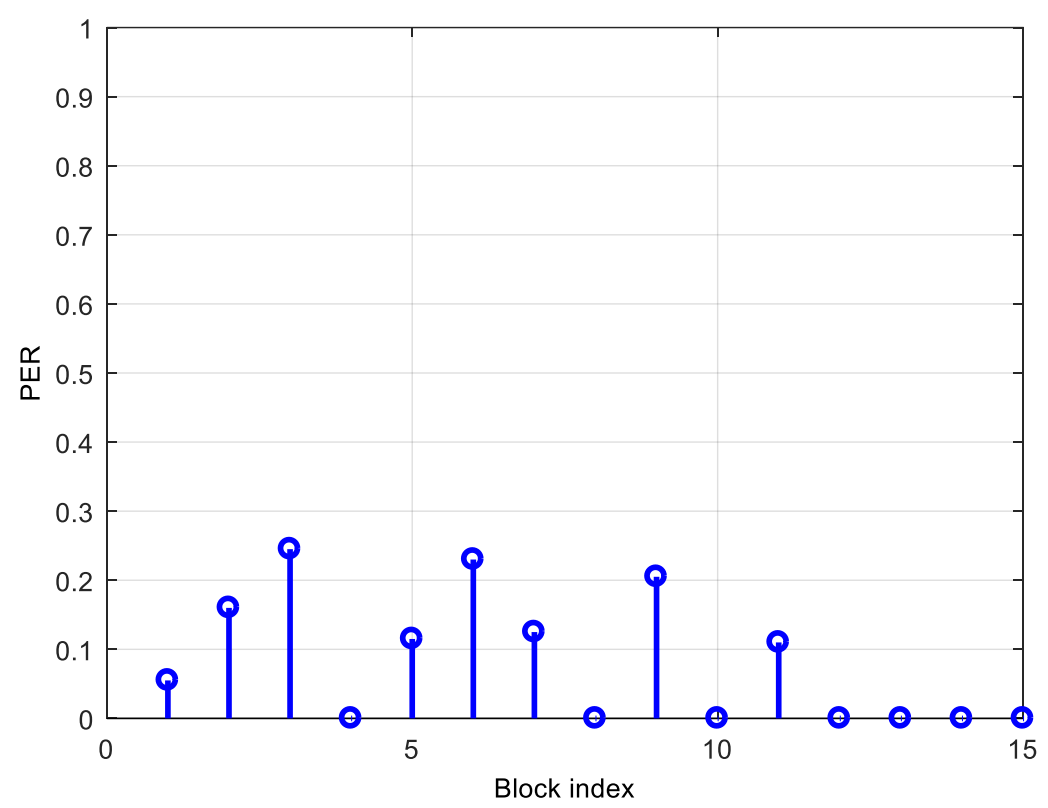

Figure 3. PER per Raptor Q source block, $K=200$

Figure 4 - Figure 6 compare the video quality in terms of PSNR for different Raptor Q source block sizes $(K=100$ and $K=200)$, Raptor Q code rates $(C R=\{0.9,0.8,0.7\})$ and $\mathrm{I}$ frame periods $\left(I_{F P}\right.$ $=5$ and $\left.I_{F P}=25\right)$. In all figures, the blue line presents the error-free PSNR values per video frame at the transmitter (BS) which is used as an upper bound in order to evaluate the quality of the video for different parameters at the receiver. Green line shows the mean PSNR which is averaged over all video frames in order to make the comparisons easier.

It is seen in Figure 4 - Figure 6 that without AL-FEC (no Raptor represents this case) it is not possible to provide error free video multicast (the mean PSNR is around $10 \mathrm{~dB}$ and $30 \mathrm{~dB}$ for I frames periods of 5 and 25 respectively). However, using AL-FEC in multicast video streaming can significantly improve the received video quality, i.e., the mean PSNR increases from $10 \mathrm{~dB}$ to $44 \mathrm{~dB}$ (up to $34 \mathrm{~dB}$ improvement in the mean PSNR depending upon the I frame period).

When different source block sizes are compared in Figure 4 and Figure 5, where the I frame period is fixed to 25, it is seen that higher source block size $(K=200)$ provided much better performance than small source block size $(K=100)$ as explained above this is due to the fact that the PER in a source decreases with the increasing source block size. For example, for $K=200$, the $C R=0.7$ can provide error free video multicasting, however, for $K=100$ it does not. It should be noted that higher $K$ causes longer delay thus it must be selected depending on the applications`QoS requirements since video applications cannot tolerate any delays and hence packets delayed beyond the applications` QoS requirements are considered as lost. 


\section{Reliable Video Multicasting over WLANs}

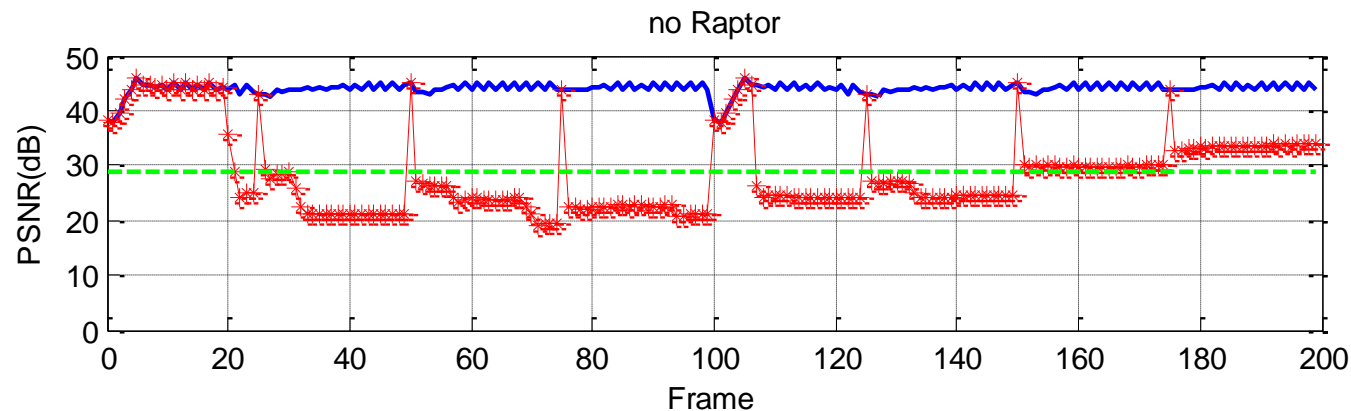

$\mathrm{CR}=0.9$

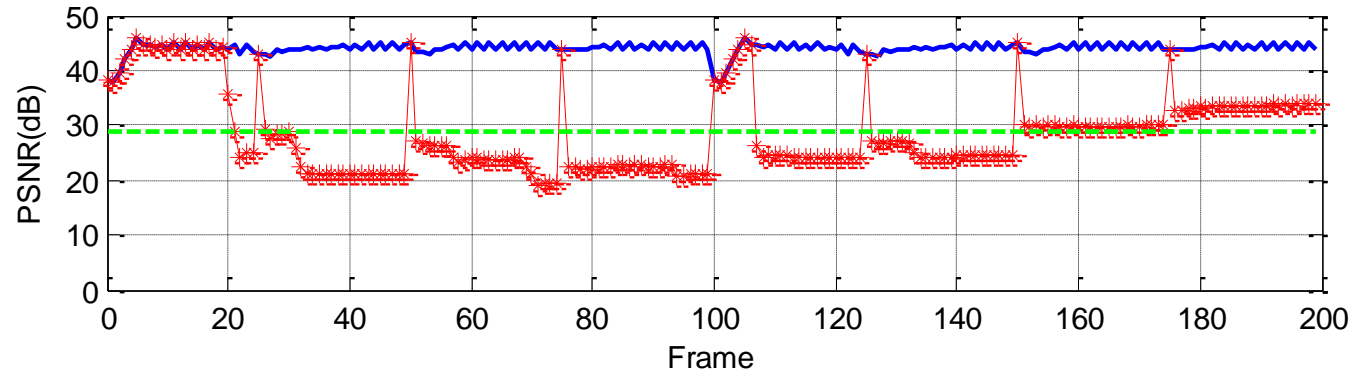

$\mathrm{CR}=0.8$

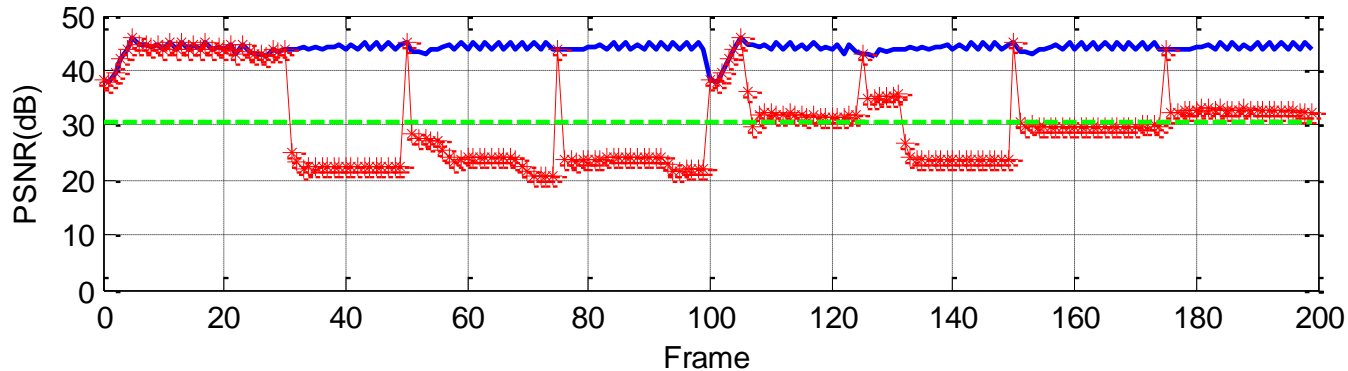

$\mathrm{CR}=0.7$

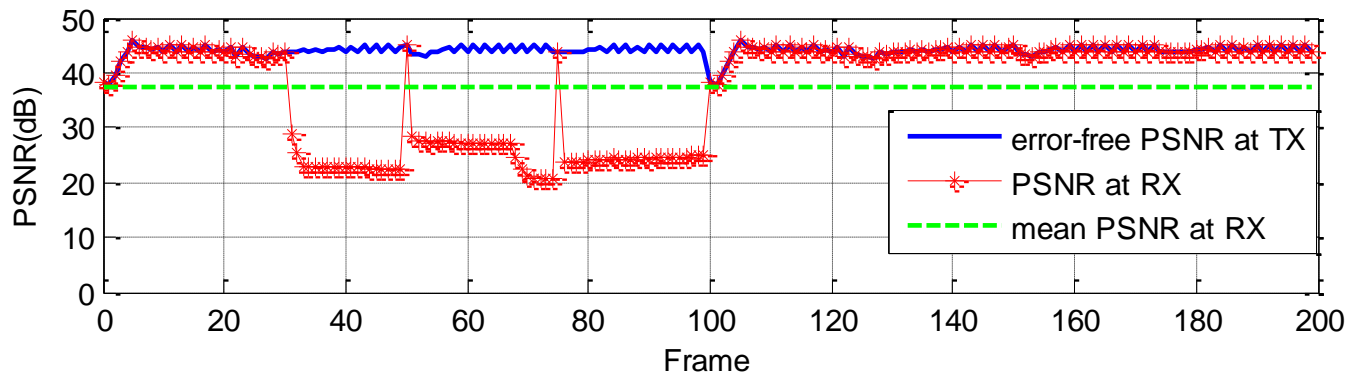

Figure 4. PSNR per frame for $K=100$, I frame period of 25

When comparing the video quality for different I frame periods, it is seen that using higher I frame period $\left(I_{F P}=25\right)$ provides higher peak and mean PSNR values. Mean PSNRs for $I_{F P}=5$ and $I_{F P}=25$ are around $10 \mathrm{~dB}$ and $30 \mathrm{~dB}$ respectively, and peak PSNR values for $I_{F P}=5$ are zero for frames of 30100 and 132-200 when no AL-FEC is implemented. For these frames when $I_{F P}=25$ is used the PSNR values are ranges between $20 \mathrm{~dB}$ to $33 \mathrm{~dB}$ but never reduced to zero. Thus, when I frame period of 25 is used the system can still provide some services unlike I frame period of 5 . As seen in Figure 5 and Figure 6 that sending I frame frequently increases the propablity of loss of the I frame. Therefore, when I frame is lost it is not possible to decode the $\mathrm{P}$ frames since $\mathrm{P}$ frames are predicted from I and $\mathrm{P}$ frames. 

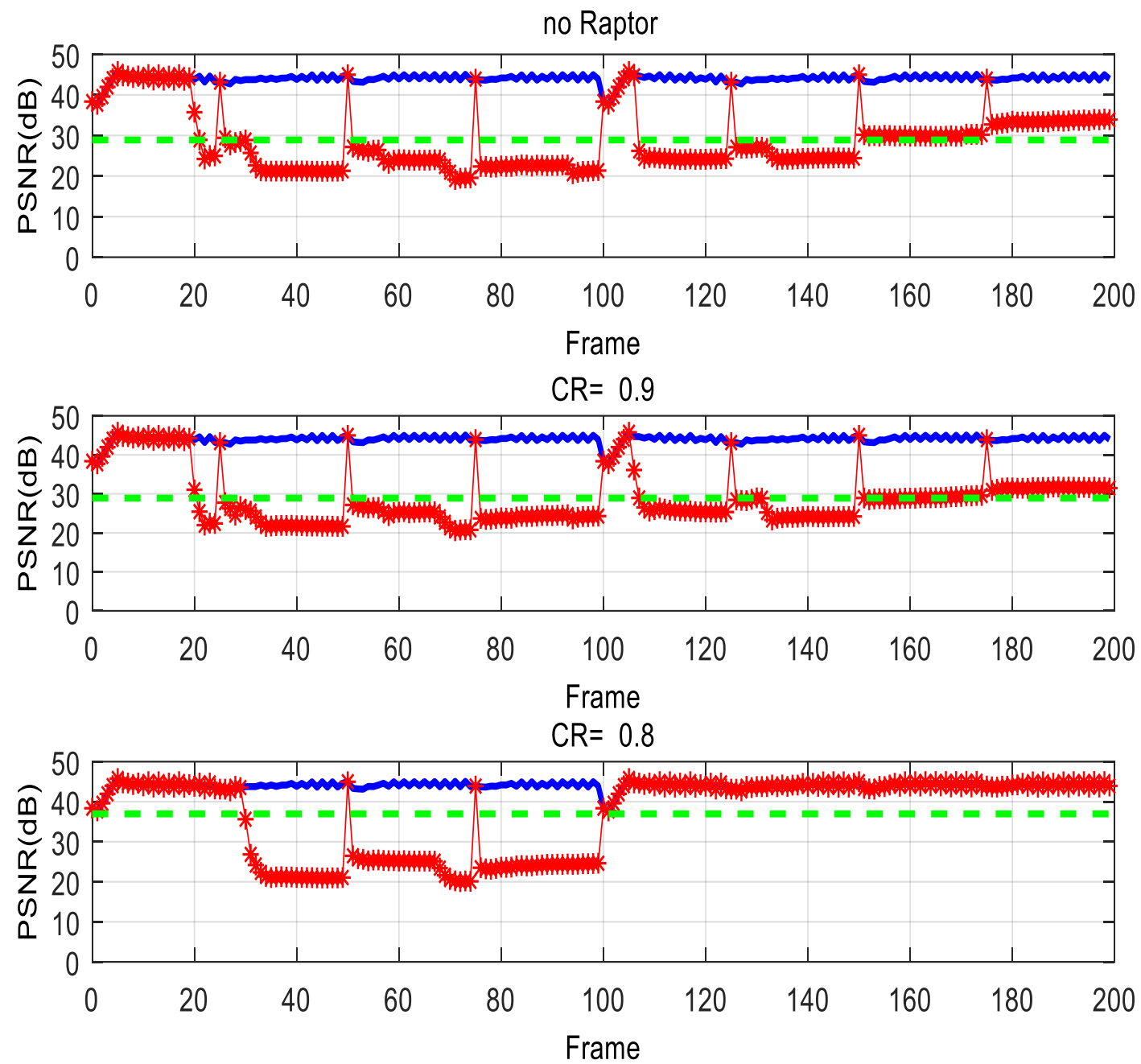

$\mathrm{CR}=0.7$

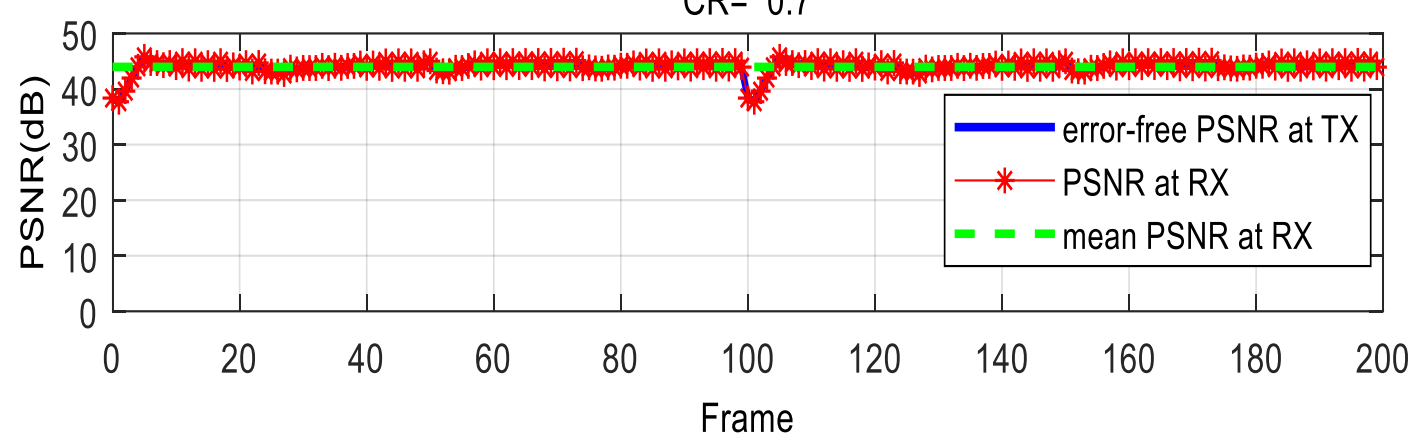

Figure 5. PSNR per frame for $K=200$, I frame period of 25

In Figure 3, it is observed that the source block index of 12-15 attacin zero PER. However, in Figure 5 the PSNR vales are ranging from $29 \mathrm{~dB}$ to $35 \mathrm{~dB}$ for the frames between 120-200. Although these frames are received error free due to the use of predictive coding, PER temporally propagates to adjacent frames and hence the calculated PSNRs are less than the reference PSNR values (blue line). These results suggest that for video multicasting the I frame must be transmitted less frequently in order to provide reliable and robust video streaming. Since, in multicast there is no feedback mechanism from the receiver, the transmitter cannot change the parameter when the video quality is poor. 


\section{Reliable Video Multicasting over WLANs}

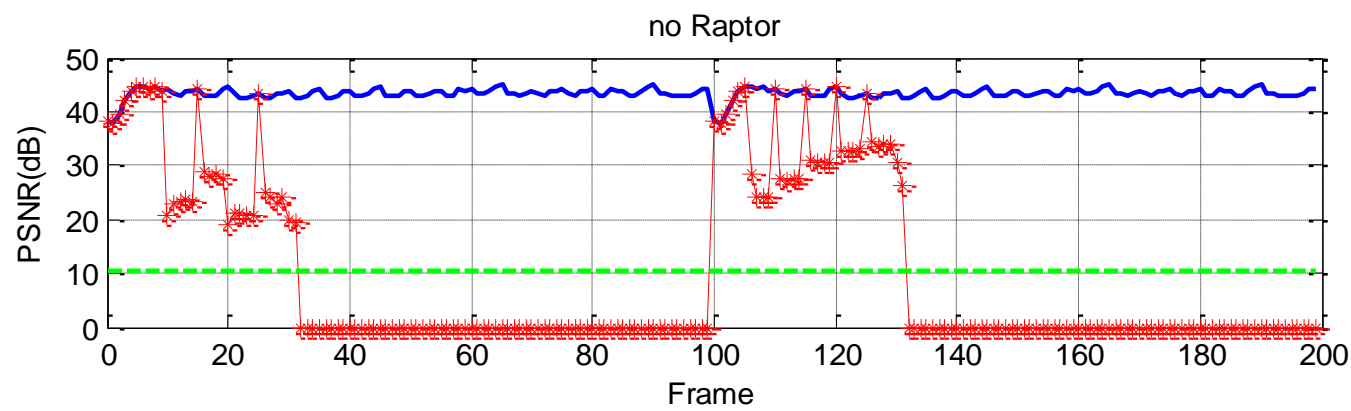

$\mathrm{CR}=0.9$

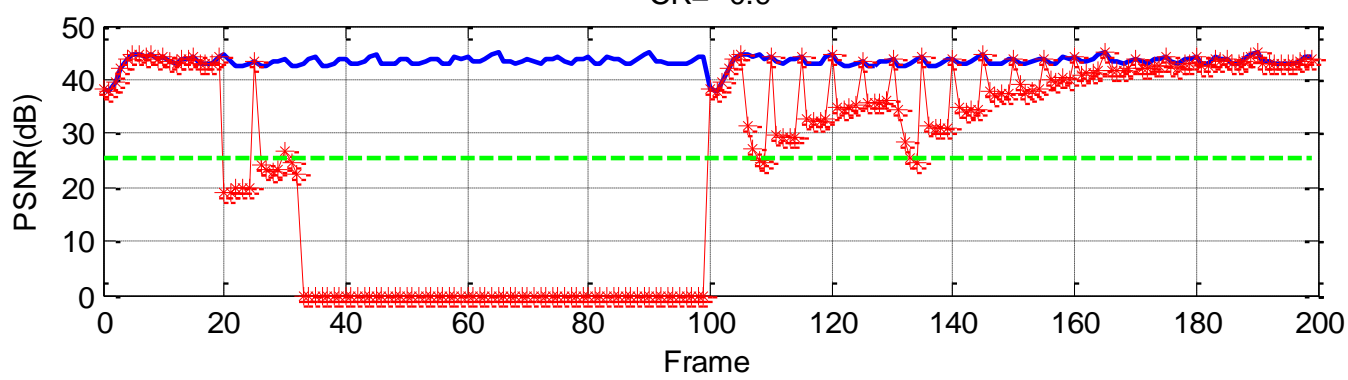

$\mathrm{CR}=0.8$

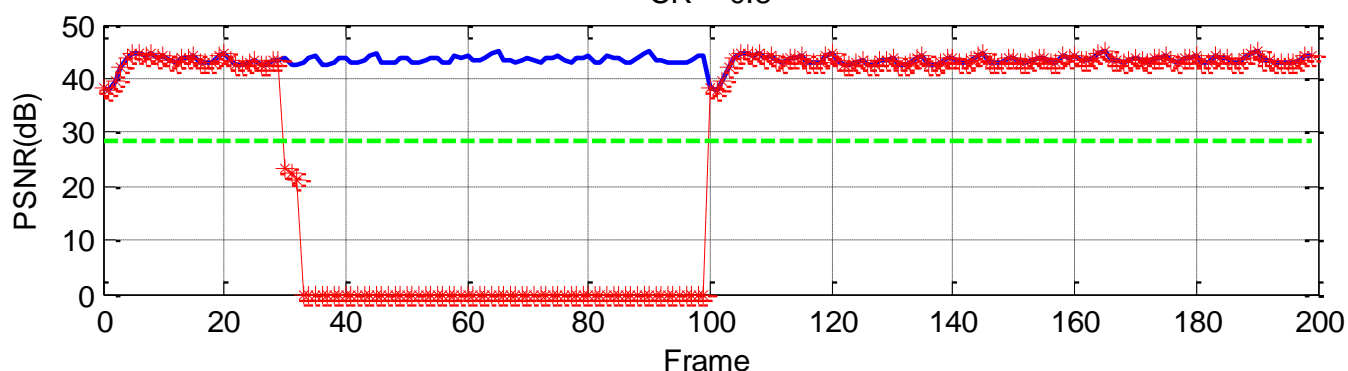

$\mathrm{CR}=0.7$

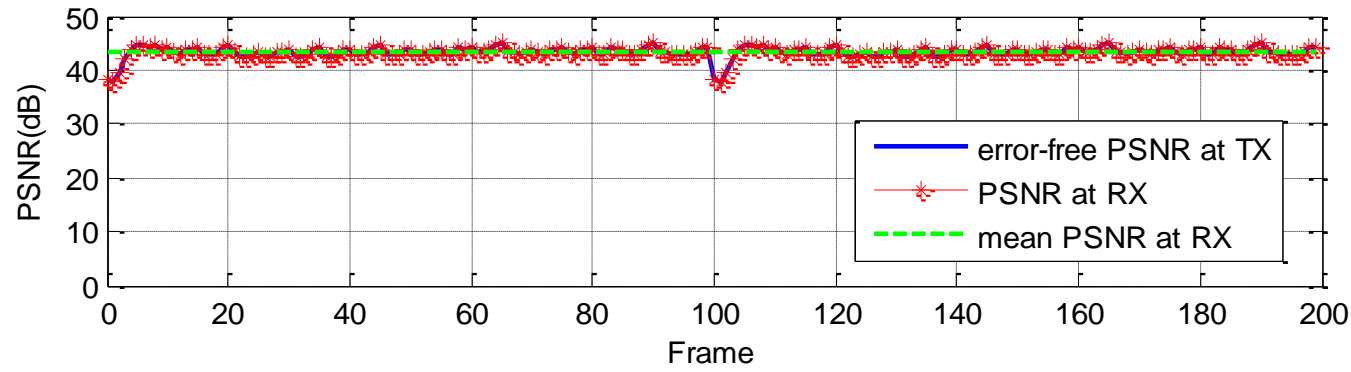

Figure 6. PSNR per frame for $K=200$, I frame period of 5

\section{CONCLUSION}

This paper presented a reliable wireless video multicast scheme over WLANs based on Raptor Q AL-FEC codes. Since different applications (video streaming or download delivery) have different QoS requirements, the system performance has to be evaluated and optimised for different parameters such as Raptor Q code rate, source block size, I frame period etc. Therefore, in this paper, an advanced andto-end system level simulator was developed to investigate the video quality in terms of PSNR for different parameters. It was shown that without Raptor Q AL-FEC codes it is not possible to provide reliable video multicasting over WLANs. Further that $34 \mathrm{~dB}$ PSNR improvement can be achieved with AL-FEC codes. To further improve the performance, different I frame periods were also compared. It was observed that inserting I frames frequently results in poor video quality (low PSNR values) therefore I frames must be transmitted at longer intervals. 


\section{REFERENCES}

3GPP TS 26.346 V8.0.0, 2008. Universal mobile telecommunications system (UMTS); multimedia broadcast/multicast service (MBMS); Protocols and codecs.

Afzal J, Stockhammer T, Gasiba T, Xu W, 2006. System design options for video broadcasting over wireless networks. 3rd IEEE Consumer Communications and Networking Conference, Las Vegas, January 8-10, 2006, pp: 938-943.

Chen MN, Lin CW, Chen YC, 2007. Adaptive error-resilience transcoding and fairness grouping for video multicast over wireless networks. IEEE Internatıonal Communications Conference ICC, Glasgow, June 2428, 2007, pp: 1661-1666.

Choi M, Sun W, Koo J, Choi, S, Shin KG, 2014. Reliable video multicast over Wi-Fi networks with coordinated multiple APs. IEEE Conference on Computer Communications, Toronto, April 27- May 2, 2014, pp: 424432.

Erceg V, Schumacher L, Kyritsi P, et al., 2004. TGn Channel Models. IEEE Tech. Rep. 802.11- 03/940r4, May 10.

Ferre P, 2006. Cross-Layer Analysis for Video Transmission over COFDM-based Wireless Local Area Networks. University of Bristol Ph.D. Thesis (Printed).

H.264/AVC reference software, 2015. http://iphome.hhi.de/suehring/tml/ (Date of access: 11 September 2015).

Haratcherev I, Taal J, Langendoen K, Lagendijk R, Sips H, 2006. Optimized Video Streaming over 802.11 by cross-layer signalling. IEEE Communications Magazine, 40(1): 115-121.

IEEE Std 802.11, 2012. International Standard for Information Technology -Telecommunications and information exchange between systems-Local and metropolitan area networks-Specific Requirements Part 11: Wireless LAN Medium Access Control (MAC) and Physical Layer (PHY) specifications.

ITU-T Recommendation H.264, 2009. Advanced Video Coding for Generic Audio visual Services. ISO/IEC 14496-10 (2009): Information Technology - Coding of Audio-visual Objects - Part 10: Advanced Video Coding.

Ma J, Feng X, Liu Y, Tang B, 2005. Video multicast over WLAN. IEEE International Symposium on Communications and Information Technology, Beijing, October 12-14, 2005, pp: 1400-1403.

Lee S, Chung K, 2008. Joint Quality and Rate Adaptation Scheme for Wireless Video Streaming. IEEE 22nd International Conference on Advanced Information Networking and Applications, Okinawa, March 25-28, 2008, pp:311-318.

Samokhina M, Suwon K, Moklyuk K, Choi S, Seoul K, Heo J, 2008. Raptor code-based video multicast over IEEE 802.11 WLAN. Conference of Asia Pacific Wireless Communications Symposium, 2008.

Shokrollahi A, 2006. Raptor codes. IEEE Transactions on Information Theory, 52(6): 2551-2567.

Shokrollahi A., Luby M, 2011. Raptor Codes. Foundations and Trends in Communications and Information Theory, 6(3), 213-322.

Stockhammer T, Hannuksela MM, Wiegand T, 2003. H.264/AVC in Wireless Environments. IEEE Transactions on Circuits and Systems for Video Technology, 13(7): 657-673.

Van der Schaar M, Turaga D, 2007. Cross-layer optimization and retransmission strategies for delay sensitive multimedia transmission. IEEE Transaction on Multimedia, 9 (1):185-197.

Wan L., Tsai S, Almgren M, 2006. A fading-insensitive performance metric for a unified link quality model. Wireless Communications and Networking Conference, Las Vegas, 3-6 April, 2006, pp: 2110-2114.

Wiegand T, Sullivan G, Bjontegaard G, Luthra A, 2003. Overview of the H.264/AVC Video Coding Standard. IEEE Transactions on Circuits and Systems for Video Technology, 13(7): 560-576. 